\title{
Implementation of Antimicrobial Stewardship Measures in Cardiac Surgery: Change of Protocol for Surgical Antibiotic Prophylaxis and Its Impact on Surgical Site Infections
}

\section{Güzin Surat ( $\nabla$ surat_g@ukw.de)}

Department of Infection Control and Antimicrobial Stewardship. University Hospital of Würzburg, JosefSchneider-Straße 2, 97080 Würzburg, Germany

\section{Dominik Bernsen}

Julius-Maximilians-Universität Würzburg: Julius-Maximilians-Universitat Wurzburg

\section{Christoph Schimmer}

Department of Cardiothoracic and Thoracic Vascular Surgery, University Hospital of Würzburg, Germany

\section{Research}

Keywords: Perioperative prophylaxis, antimicrobial stewardship, cardiac surgery: cefazolin, cefuroxime

Posted Date: September 25th, 2020

DOI: https://doi.org/10.21203/rs.3.rs-80948/v1

License: (c) (1) This work is licensed under a Creative Commons Attribution 4.0 International License.

Read Full License 
Implementation of antimicrobial stewardship measures in cardiac surgery: Change of protocol for surgical antibiotic prophylaxis and its impact on surgical site infections

G. Surat ${ }^{1 *}$, D. Bernsen ${ }^{2}$, C. Schimmer ${ }^{3}$

*Correspondence: surat g@ukw.de

${ }^{1}$ Department of Infection Control and Antimicrobial Stewardship.

University Hospital of Würzburg, Josef-Schneider-Straße 2, 97080 Würzburg, Germany

Full list of author information is available at the end of the article 


\section{Abstract \\ Objectives:}

The goal of this study was to evaluate the impact on a protocol change of surgical antimicrobial prophylaxis in cardiac surgery comparing the new in hospital prophylactic agent cefazolin versus the former standard antibiotic cefuroxime, initiated by the antimicrobial stewardship team.

\section{Methods:}

This retrospective observational study was performed in a tertiary care hospital in collaboration with the department of cardiothoracic surgery and the antimicrobial stewardship team following a new recommendation of the surgical antimicrobial prophylaxis and included 1029 patients who underwent cardiac surgery.

Two patient groups including 582 patients receiving Cefuroxime and 447 patients receiving Cefazolin respectively were compared without altering any other preventative perioperative measures. Adherence, overall infection rates, leg wound infections and deep sternal wound infections were compiled and analysed as well as a broad profile of preoperative, intraoperative and postoperative patient data.

\section{Results:}

A complete adherence on the change of the agent was achieved.

Overall surgical site infections occurred in 37 (6.6\%) of the cases, with $20(3,4 \%)$ SSI in cefuroxime patients and $17(3,8 \%)$ in cefazolin patients ( $p$-value=0,754).

No statistically significant differences could be found in any of the primary and secondary endpoints including DSWI (2,1\% for cefuroxime, 1,3\% for cefazolin), vein harvesting site infections (1,2\% for cefuroxime, 2,2\% for cefazolin) as well as urinary tract infections, pneumonia, sepsis and overall mortality of all causes.

\section{Conclusions:}

These results demonstrate that compliance to antimicrobial stewardship standards is not associated with more surgical site infections.

\section{Keywords:}

Perioperative prophylaxis; antimicrobial stewardship; cardiac surgery: cefazolin; cefuroxime 


\section{Introduction}

Antibiotic prophylaxis in open-heart surgery has been an established procedure for decades. Regarding the invasiveness and mortality risks of postoperative sternal and mediastinal wound infections optimal antibiotic prophylaxis is crucial. Yet the recommended antibiotic regimen has changed many times over the decades and still varies with each center.

The main principles of perioperative antimicrobial prophylaxis include the timing, dosage and duration as well as the choice of the given agent. The most recommended choice of agents for cardiac procedures belong to the class of cephalosporins. The majority of recommendations regarding surgical prophylaxis for cardiothoracic operations is based on studies and findings from the 1990s showing that the firstgeneration cephalosporin cefazolin is as effective as second-generation cephalosporins like cefamandole or cefuroxime for the prevention of surgical site

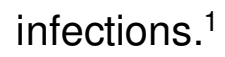

A more recent study by Sommerstein et al. implies that Cefazolin might be the better agent for surgical antibiotic prophylaxis in cardiac surgery for its use reduced significantly the rate of overall surgical site infections when compared to cefuroxime. ${ }^{2}$ The university hospital of Würzburg (UKW) in Bavaria/Germany, launched an antimicrobial stewardship program (ASP) in July 2015 complying with the German Act on Prevention and Control of Infectious Diseases (Infektionsschutzgesetz §23) as response to the medical crisis due to increasing antimicrobial resistance. ${ }^{3}$ Antimicrobial stewardship programs are developed with the goal to improve the quality of antimicrobial use and to sustain the efficacy of antimicrobial drugs in order to improve patient outcomes. ${ }^{4,5}$ In accordance to national and international guidelines core elements of antimicrobial stewardship were implemented and in its wake antibiotic ward rounds and antimicrobial prescribing guidelines introduced. ${ }^{5,6}$ The existing inhospital guideline on antimicrobial surgical prophylaxis was updated and amended in May 2017 affecting a dozen of surgical departments, favoring cefazolin as the new routine agent instead of the thitherto used cefuroxime.

The main objective of this retrospective study was to monitor the change and to scan its impact on adherence, surgical site infections, nosocomial infections and risk factors. 
The study reflects a multidisciplinary approach on improving the quality of antimicrobial use and was conducted in collaboration with the department of cardiothoracic surgery and the antimicrobial stewardship team of the UKW.

\section{Methods}

\section{Study design}

This retrospective analysis involved 1029 inpatients who underwent coronary artery bypass grafting and/or valve surgery via median sternotomy admitted between July 30th, 2016 and December 27th, 2017 at the department of cardiothoracic surgery of the UKW.

Patients who had preoperatively ongoing thoracic infections, were diagnosed with endocarditis or osteitis in two or more locations, patients who underwent heart transplantation or pregnant and breastfeeding women were excluded.

In line with the hospital new guideline on the use of surgical antimicrobial prophylaxis, launched May 2017, every patient received cefazolin $2 \mathrm{~g}$, instead previously cefuroxime $1.5 \mathrm{~g}$. There was no change in the duration of the perioperative prophylaxis for open-heart surgery for a maximum of 3 applications, administering the first dose preoperatively shortly (ideally 30 minutes) before surgical incision. Any prolongation of surgery ( $>2$ half-lives of the prophylactic agent) required a second dose intraoperatively, completing the perioperative prophylaxis with a final dose 3-4 hours after the last preceding dose. We compared 582 patients (group 1) who previously had received cefuroxime compared to 447 patients (group 2) who received Cefazolin in accordance to the new protocol.

\section{Data collection}

Data collection was performed from the patient's files. All medical records were processed and analysed anonymously, so an ethics votum was not needed.

The data collection involved patient's preoperative status, the intraoperative course and the postoperative progress. The research followed a standardized survey sheet for every patient. 


\section{Definitions}

All patients were checked on a daily basis, especially on the progress of wound healing or any other signs of infections. Additionally, to the physical assessments full blood panels were performed.

The applied end points of this study were carried out in compliance to the CDCcriteria. ${ }^{7}$ The primary endpoints in this study were overall surgical site infections (SSI), deep sternal wound infections (DSWI) and infections of the vein harvesting site. Secondary endpoints were defined as pneumonia or tracheobronchitis, urinary tract infections, sepsis and death of all causes.

\section{Statistical analysis}

The statistical analysis was performed using SPSS. A $p$ value of $\leq 0,05$ was defined as statistically significant. A descriptive overview over both groups was given and then analysed using the Mann-Whitney $U$ Test for continuous variables and chi-squared test for categorical variables. This was done in order to determine statistical differences between the two groups. Furthermore the primary and secondary endpoints were analysed using Chi-Square test and Mann-Whitney $U$ test according to the scale type. Sub-group analyses were performed using the same tests for univariate analysis. A multiple logistic regression analysis was conducted subsequently to test and confirm the independency of the risk factors.

\section{Results}

1029 patients could be included in the study. 582 (56.6\%) patients received cefuroxime and 447 (43.4\%) cefazolin as the prophylactic antibiotic. All patients were screened for preoperative characteristics (table 1), comorbidities, intraoperative data and postoperative variables and treatments. Some significant statistical differences between the two patient groups could be found as for preoperative data showed differences in creatinin and leukocyte levels as well as fewer smokers in the cefuroxime group. Furthermore differences in the intubation period, the drainage flow rate and the number of postoperative delirium were found analyzing postoperative variables. From the clinical point of view these differences can be interpreted as non- significant. Statistical tests indicate a significance because of the large study population even 
when the study groups practically show little to no difference (Table 1-3 in Supplement).

\begin{tabular}{|c|c|c|c|c|c|}
\hline & & Total & Cefuroxime & Cefazolin & $\mathbf{p}$ \\
\hline \multicolumn{2}{|c|}{ Patient number (\%) } & $1029(100)$ & $582(56,6)$ & $447(43,4)$ & \\
\hline \multirow[t]{2}{*}{$\operatorname{Sex}(\%)$} & m & $782(76,0)$ & $435(74,7)$ & $347(77,6)$ & \multirow[t]{2}{*}{0,283} \\
\hline & f & $247(24,0)$ & $147(25,3)$ & $100(22,4)$ & \\
\hline \multicolumn{2}{|l|}{ Age (Years) } & $68,2 \pm 9,86$ & $68,0 \pm 10,11$ & $68,48 \pm 9,51$ & 0,640 \\
\hline \multicolumn{2}{|l|}{ BMI $\left(\mathrm{kg} / \mathrm{m}^{2}\right)$} & $28,72 \pm 4,73$ & $28,90 \pm 4,68$ & $28,48 \pm 4,80$ & 0,162 \\
\hline \multirow{5}{*}{ BMI Grad (\%) } & \multirow{5}{*}{$\begin{array}{r}\text { Normal weight } \\
\text { Overweight } \\
\text { Class I Obesity } \\
\text { Class II Obesity } \\
\text { Class III Obesity }\end{array}$} & $205(21,3)$ & $113(20,7)$ & $92(22,0)$ & \multirow{5}{*}{0,673} \\
\hline & & $419(43,5)$ & $230(42,2)$ & $189(45,1)$ & \\
\hline & & $247(25,6)$ & $149(27,3)$ & $98(23,4)$ & \\
\hline & & $72(7,5)$ & $40(7,3)$ & $32(7,6)$ & \\
\hline & & $21(2,2)$ & $13(2,4)$ & $8(1,9)$ & \\
\hline \multicolumn{2}{|c|}{ Creatinin $(\mathrm{mg} / \mathrm{dl})$} & $1,32 \pm 3,10$ & $1,35 \pm 4,02$ & $1,28 \pm 1,07$ & 0,037 \\
\hline \multicolumn{2}{|l|}{ MDRD } & $72,0 \pm 25,56$ & $72,9 \pm 24,86$ & $70,9 \pm 26,43$ & 0,092 \\
\hline \multicolumn{2}{|c|}{ Leucocytes (1/nl) } & $8,09 \pm 3,90$ & $7,93 \pm 4,51$ & $8,30 \pm 2,90$ & 0,009 \\
\hline \multicolumn{2}{|l|}{ CRP (mg/l) } & $0,81 \pm 2,22$ & $0,73 \pm 1,73$ & $0,92 \pm 2,73$ & 0,578 \\
\hline \multicolumn{2}{|l|}{ HbA1c (\%) } & $6,00 \pm 1,00$ & $6,01 \pm 0,99$ & $5,98 \pm 1,01$ & 0,296 \\
\hline
\end{tabular}

Table 1. Preoperative patient data

Regarding the endpoints (table 2) of this study no statistically significant differences between both study groups could be found. Overall 37 (3,6\%) patients suffered some form of surgical site infection (SSI), including 18 (1,7\%) deep sternal wound infections, $17(1,7 \%)$ documented infections of the vein harvesting site. $20(3,4 \%)$ surgical site infections occurred in the cefuroxime group compared to $17(3,8 \%)$ in the cefazolin group ( $p=0,754)$. Deep sternal wound infections could be diagnosed in $12(2,1 \%)$ of the cefuroxime patients and $6(1,3 \%)$ of cefazolin patients $(p=0,383)$. Regarding secondary endpoints the two antibiotics didn't demonstrate any statistically significant differences either. Overall $40(4,0 \%)$ urinary tract infections, 70 (7,0\%) lower 
respiratory tract infections and $11(1,1 \%)$ cases of sepsis occurred in both groups. The overall mortality was $3,9 \%$ with $3,3 \%$ in the cefuroxime group an $4,7 \%$ in the cefazolin group $(p=0,238)$.

\begin{tabular}{|l|c|c|c|c|}
\hline \multicolumn{1}{|c|}{ Endpoints of the study } & Total & Cefuroxime & Cefazolin & p \\
\hline SSI total (\%) & $37(3,6)$ & $20(3,4)$ & $17(3,8)$ & 0,754 \\
\cline { 2 - 5 } DSWI (\%) & $18(1,7)$ & $12(2,1)$ & $6(1,3)$ & 0,383 \\
\cline { 2 - 5 } Vein harvesting site (\%) & $17(1,7)$ & $7(1,2)$ & $10(2,2)$ & 0,197 \\
\hline Urinary tract infections (\%) & $40(4,0)$ & $26(4,6)$ & $14(3,3)$ & 0,306 \\
\hline $\begin{array}{l}\text { Pneumonia/trachebronchitis } \\
(\%)\end{array}$ & $70(7,0)$ & $41(7,1)$ & $29(6,7)$ & 0,818 \\
\hline Sepsis (\%) & $11(1,1)$ & $8(1,4)$ & $3(0,7)$ & 0,294 \\
\hline Death (\%) & $40(3,9)$ & $19(3,3)$ & $21(4,7)$ & 0,238 \\
\hline
\end{tabular}

Table 2. Primary and secondary endpoints of the study

Furthermore we were able to determine risk groups within the entire study population for developing surgical site infections in general and deep sternal wound infections (table 3). Univariate analyses showed significant risk groups were CRP $\geq 1 \mathrm{mg} / \mathrm{dl}$ $(p=0,005)$, peripheral arterial disease $(p=0,009)$, renal insufficiency $(p=0,023)$, operating time $(p=0,000)$, revision operation $(p=0,000)$, myocardial infarction within half a year before surgery $(p=0,027)$, transfusions $\geq 5 \quad(p=0,000)$ and the duration of intubation $(p=0,031)$. 


\begin{tabular}{|c|c|c|c|c|c|}
\hline \multicolumn{2}{|l|}{ Infections } & SSI & p & DSWI & p \\
\hline \multirow{2}{*}{$\begin{array}{l}\text { Age (\% within } \\
\text { subgroup) }\end{array}$} & $\geq 80 a$ & $3(2,9)$ & 0,668 & $2(1,9)$ & 0,898 \\
\hline & $<80 a$ & $34(3,7)$ & & $16(1,7)$ & \\
\hline \multirow[t]{2}{*}{$\operatorname{Sex}(\%)$} & $\mathrm{m}$ & $25(3,2)$ & 0,221 & $12(1,5)$ & 0,350 \\
\hline & $f$ & $12(4,9)$ & & $6(2,4)$ & \\
\hline \multirow[t]{2}{*}{ BMI (\%) } & $\geq 30 \mathrm{~kg} / \mathrm{m}^{2}$ & $16(4,7)$ & 0,179 & $9(2,6)$ & 0,123 \\
\hline & $<30 \mathrm{~kg} / \mathrm{m}^{2}$ & $21(3,0)$ & & $9(1,3)$ & \\
\hline \multirow[t]{2}{*}{ CRP (\%) } & $\geq 1 \mathrm{mg} / \mathrm{dl}$ & $12(7,4)$ & 0,005 & $6(3,7)$ & 0,040 \\
\hline & $<1 \mathrm{mg} / \mathrm{dl}$ & $25(2,9)$ & & $12(1,4)$ & \\
\hline \multirow[t]{2}{*}{ Leucocytes (\%) } & $\geq 9 / \mathrm{nl}$ & $12(4,6)$ & 0,322 & $6(2,3)$ & 0,439 \\
\hline & $<9 / \mathrm{nl}$ & $25(3,3)$ & & $12(1,6)$ & \\
\hline \multirow[t]{2}{*}{ COPD (\%) } & yes & $5(5,3)$ & 0,348 & $3(3,2)$ & 0,264 \\
\hline & no & $32(3,4)$ & & $15(1,5)$ & \\
\hline \multirow[t]{2}{*}{ PAD (\%) } & yes & $8(8,3)$ & 0,009 & $3(3,1)$ & 0,280 \\
\hline & nein & $29(3,1)$ & & $15(1,6)$ & \\
\hline \multirow{2}{*}{$\begin{array}{l}\text { Diabetes mellitus } \\
\text { (\%) }\end{array}$} & yes & $15(4,6)$ & 0,227 & $7(2,2)$ & 0,495 \\
\hline & no & $22(3,1)$ & & $11(1,6)$ & \\
\hline \multirow{2}{*}{$\begin{array}{l}\text { Nicotine abuse } \\
\text { (\%) }\end{array}$} & yes & $15(4,3)$ & 0,357 & $6(1,7)$ & 0,986 \\
\hline & no & $22(3,2)$ & & $12(1,8)$ & \\
\hline \multirow{2}{*}{$\begin{array}{l}\text { Myocardial } \\
\text { infarction within the } \\
\text { past half year (\%) }\end{array}$} & yes & $12(5,1)$ & 0,152 & $8(3,4)$ & 0,027 \\
\hline & no & $25(3,1)$ & & $10(1,3)$ & \\
\hline \multirow{2}{*}{$\begin{array}{l}\text { Ejection fraction } \\
<30 \%(\%)\end{array}$} & yes & $2(4,2)$ & 0,828 & $1(2,1)$ & 0,857 \\
\hline & no & $35(3,6)$ & & $17(1,7)$ & \\
\hline \multirow{2}{*}{$\begin{array}{l}\text { Renal failure } \\
(\%)\end{array}$} & yes & $12(6,4)$ & 0,023 & $7(3,7)$ & 0,022 \\
\hline & no & $25(3,0)$ & & $11(1,3)$ & \\
\hline \multirow[t]{2}{*}{ Emergency (\%) } & yes & $5(3,1)$ & 0,704 & $3(1,9)$ & 0,914 \\
\hline & no & $32(3,7)$ & & $15(1,7)$ & \\
\hline Incision/Suture & $\geq 240 \mathrm{~min}$ & $26(7,2)$ & 0,000 & $11(3,0)$ & 0,021 \\
\hline
\end{tabular}




\begin{tabular}{|l|r||c|c|c|c|}
\hline time (\%) & $<240 \mathrm{~min}$ & $11(1,7)$ & & $7(1,1)$ & \\
\hline HLM time (\%) & $\geq 90 \mathrm{~min}$ & $30(4,1)$ & 0,182 & $14(1,9)$ & 0,542 \\
\hline X-Clamp time (\%) & $\geq 60 \mathrm{~min}$ & $7(2,4)$ & & $4(1,4)$ & \\
\hline & $<60 \mathrm{~min}$ & $31(4,1)$ & 0,173 & $14(1,8)$ & 0,723 \\
\hline Revision surgery & yes & $23(2,3)$ & & $4(1,5)$ & \\
\hline (\%) & no & $14(1,5)$ & & $0(0,0)$ & \\
\hline Transfusions (\%) & $\geq 5$ & $14(11,8)$ & 0,000 & $10(8,4)$ & 0,000 \\
\hline Duration of & $<5$ & $23(2,5)$ & & $8(0,9)$ & \\
\hline intubation (\%) & $\geq 24 \mathrm{~h}$ & $9(5,7)$ & 0,118 & $6(3,8)$ & 0,031 \\
\hline
\end{tabular}

Table 3. Univariate analysis of patient risk groups

The significant risk factors from the univariate analysis were included in a multiple logistic regression analysis (table 4). Four risk factors could be confirmed as independent predictors for surgical site infections including CRP $\geq 1 \mathrm{mg} / \mathrm{dl} \quad(p=0,013)$, peripheral arterial disease $(p=0,039)$, operating time $(p=0,007)$ and revision operation $(p=0,000)$.

\begin{tabular}{|l|c|c|c|c|c|c|c|}
\hline & B & SD & Wald & p & OR & 95\% & CI \\
\hline CRP & $-0,15$ & 0,06 & 6,11 & 0,013 & 0,857 & 0,759 & 0,969 \\
\hline PAD & 1,11 & 0,54 & 4,26 & 0,039 & 30,28 & 1,058 & 8,666 \\
\hline $\begin{array}{l}\text { Myocardial infarction } \\
\text { within the past half } \\
\text { year }\end{array}$ & $-0,11$ & 0,49 & 0,05 & 0,824 & 0,896 & 0,341 & 2,357 \\
\hline Renal failure & 0,56 & 0,46 & 1,45 & 0,228 & 1,749 & 0,705 & 4,337 \\
\hline Incision/suture time & $-0,01$ & 0,003 & 7,31 & 0,007 & 0,993 & 0,987 & 0,998 \\
\hline Revision surgery & 3,42 & 0,45 & 56,66 & 0,000 & 30,536 & 12,537 & 74,377 \\
\hline Total of transfusions & $-0,01$ & 0,04 & 0,16 & 0,690 & 0,986 & 0,919 & 1,058 \\
\hline Duration of intubation & 0,01 & 0,01 & 1,77 & 0,183 & 1,007 & 0,997 & 1,016 \\
\hline Constant & $-3,77$ & 1,69 & 4,98 & 0,026 & 0,023 & & \\
\hline
\end{tabular}


Table 4. Multiple logistic regression analysis of risk groups

$B=$ coefficient of the logistic regression, $S D=$ standard deviation, Wald=Wald-test, $p=p$-value, $\mathrm{OR}=$ Odds ratio, $\mathrm{Cl}=$ Confidence interval

Within the previously identified risk groups no statistically significant difference between the cefuroxime and cefazolin group regarding surgical site infections in total and deep sternal wound infections could be evaluated (Table 4 in Supplement). In all cases of deep sternal wound infections and mediastinitis microbiological analyses were performed to diagnose specific bacteria responsible for the infections. Staphylococcus epidermidis and Staphylococcus aureus were the leading causes for the infections and were found in 10 cases (Table 5 in Supplement).

\section{Discussion}

The right antibiotic choice for reducing the risk of postoperative sternal infections and mediastinitis in cardiac surgery has been subject to a large number of studies in the last decades. Since placebo-controlled trials have demonstrated a significant advantage towards antimicrobial prophylaxis for the prevention of surgical site infections the administration of antibiotics before incision has become an obligate standard. These studies mainly originate in the 1970s and prove beta-lactamase antibiotics to be the preferred class of antibiotics for this kind of indication. ${ }^{8,9}$

While meta-analyses indicate that cephalosporins are superior to glycopeptides when it comes to overall chest infections with no resistant organisms ${ }^{10}$, the question of the right generation cephalosporin remains unproven.

One of the major reasons for that is the lack of new studies. The majority of literature on the topic dates back to the 1990s and even modern-day guidelines still base their recommendations on studies from that time. ${ }^{11}$

Different generations of cephalosporins showed no apparent differences but the trend should go towards earlier-generation cephalosporins taking the most responsible organism Staphyloccocus aureus into account. Page et al. suggest a use of cefazolin in standard cases. ${ }^{12}$ Dellinger et al. as well as Mangram et al. consent to a overall recommendation of cefazolin for everyday use in standard cases. ${ }^{13,14}$

The latest study on the topic has been performed by Sommerstein et al. ${ }^{2}$ The 2019 prospective cohort study was able to include 14 centers all over Switzerland enrolling 
a total of 21.007 patients undergoing cardiac surgery. The study was overviewed and controlled by the 'Swissnoso' center for infection surveillance. Regarding the choice of the antimicrobial substance cefazolin was compared to cefuroxime and a combination of vancomycin and cefuroxime. The overall rates for surgical site infections ranged between $4.5 \%$ to $6.1 \%$ whereas deep sternal wound infections occurred in $1.8 \%$ to $2.9 \%$ of the cases. For all surgical site infections cefazolin showed a significant reduction of infection rates, for deep infections cefazolin and the combination of vancomycin and cefuroxime achieved significantly better results than cefuroxime. In every case the second-generation cephalosporin had statistically significant higher infection rates.

Not only from the antimicrobial stewardship point of view second-generation cephalosporins should only be used if a slightly more gramnegative coverage is needed. As the use of antibiotics for surgical prophylaxis is mainly safe and clearly benefits the patient the antibiotic selection pressure is not to be underestimated, especially in the context of the well-known documented problem of prolonging antibiotic prophylaxis far beyond surgery. ${ }^{15,16}$

In order to optimize the antibiotic consumption one of the strategies initiated by our antimicrobial stewardship team was the implementation of hospital guidelines on the use of antibiotics for the prophylaxis and treatment of infections considering the hospital's antimicrobial surveillance data. The harmonization of the antimicrobial prophylaxis for surgical procedures included not only the choice of agent but also the restriction on the postoperative duration (depending on the type of surgery).

Whereas the change of the type of drug was completely met with acceptance, we reached an adherence of $100 \%$, the change of behavior with regard to prolonging surgical antibiotic prophylaxis after wound closure was not that easily accomplished and caused an uneasiness amongst all surgeons.

First antimicrobial stewardship programs were introduced more than 30 years ago and its rationale is still more than evident as antibiotic resistance continues to spread.

Surgeons have a paramount role in antimicrobial stewardship and can contribute to prescribe antibiotics in a prudent manner. ${ }^{17,18}$

We know ASP works but we also know that changing behavior remains a challenge. 19,20,21

Regarding all guidelines, meta-analyses, the international literature and the results of this retrospective observational study we can reason that a change of antimicrobial 
choice from Cefuroxime to Cefazolin as a perioperative antibiotic prophylaxis in cardiac surgery might not increase the rates of overall wound infections, including deep sternal wound infections as well as leg infections.

The most important limitation to this study is the retrospective study design. The followup time included only the admission period. Wound infections developed postdischarge were only included and evaluated if the patients presented themselves and were treated in the same surgical center. Another disadvantage of the retrospective design is that diagnostical criteria has not been defined beforehand, including all patient data.

\section{Declarations}

\section{Ethics approval and consent to participate}

This is a quality improvement study and ethics committee approval was not required.

\section{Consent for publication}

Not applicable.

\section{Availability of data and materials}

All data generated or analyzed during this study are included in this published article (and its supplementary information files).

\section{Competing interests}

The authors declare that they have no competing interests.

\section{Funding}

None.

\section{Authors' contributions}

DB and CS contributed to study design, data acquisition and analysis and interpretation of data. GS contributed to analysis and interpretation of data. GS and DB contributed to writing the manuscript. All authors read and approved the final manuscript.

\section{Acknowledgments}

Not applicable.

\section{Author details}

1Department of Infection Control and Antimicrobial Stewardship, University Hospital of Würzburg, Würzburg, Germany 2Julius-Maximilians-University Würzburg, Würzburg, Germany

${ }^{3}$ Department of Cardiothoracic and Thoracic Vascular Surgery, University Hospital of Würzburg, Würzburg, Germany 


\section{References}

1 Maki DG, Bohn MJ, Stolz SM, Kroncke GM, Acher CW, Myerowitz DP.

Comparative study of cefazolin, cefaman- dole, and vancomycin for surgical prophylaxis in cardiac and vascular operations. A double-blind randomized trial. J Thorac Cardiovasc Surg 1992;104:1423-34.

2 Sommerstein R, Atkinson A, Kuster SP, et al. Antimicrobial prophylaxis and the prevention of surgical site infection in cardiac surgery: an analysis of 21007 patients in Switzerlanddagger. Eur J Cardiothorac Surg. 2019;56(4):800-806.

3 Bundesgesetzblatt. Gesetz zur Änderung des Infektionsschutzgesetzes und weiterer Gesetze. July 28, 2011;

Infektionsschutzgesetz IfSG §23

4 Owens RC Jr. Antimicrobial stewardship: concepts and strategies in the 21st century. Diagn Microbiol Infect Dis. 2008;61(1):110-128

5 Barlam TF, Cosgrove SE, Abbo LM, et al. Implementing an Antibiotic Stewardship Program: Guidelines by the Infectious Diseases Society of America and the Society for Healthcare Epidemiology of America. Clin Infect Dis. 2016;62(10):e51-e77. 6 de With K, Wilke K, Kern WV, et al. AWMF-S3-Leitlinie Strategien zur Sicherung rationaler Antibiotika-Anwendung im Krankenhaus. January 31, 2019;

https://www.awmf.org/leitlinien/detail/l//092-001.html. Accessed March 5, 2019.

7 Nationales Referenzzentrum für Surveillance von nosokomialen Infektionen, Robert-Koch-Institut. Definitionen nosokomialer Infektionen für die Surveillance im Krankenhaus-Infektions-Surveillance-System (Kiss-Definitionen), Berlin 2017 8 Fong IW, Baker CB, McKee DC. The value of prophylactic antibiotics in aoratcoronary bypass operations: a double-blind randomized trial. J Thorac Cardiovasc Surg.

9 Austin TW, Coles JC, Burnett R, Goldbach M. Aortocoronary bypass procedures and sternotomy infections: a study of antistaphylococcal prophylaxis. Can J Surg. 1980;23(5):483-485.

10 Bolon MK, Morlote M, Weber SG, Koplan B, Carmeli Y, Wright SB. Glycopeptides are no more effective than beta-lactam agents for prevention of surgical site infection after cardiac surgery: a meta-analysis. Clin Infect Dis. 2004;38(10):1357-1363.

11Ban KA, Minei JP, Laronga C, et al. American College of Surgeons and Surgical Infection Society: Surgical Site Infection Guidelines, 2016 Update. J Am Coll Surg. 2017;224(1):59-74

12 Page CP, Bohnen JM, Fletcher JR, McManus AT, Solomkin JS, Wittmann DH. Antimicrobial prophylaxis for surgical wounds. Guidelines for clinical care [published correction appears in Arch Surg 1993 Apr;128(4):410]. Arch Surg. 1993;128(1):79_ 88.

13 Dellinger EP, Gross PA, Barrett TL, et al. Quality standard for antimicrobial prophylaxis in surgical procedures. Infectious Diseases Society of America. Clin Infect Dis. 1994;18(3):422-427.

14 Mangram AJ, Horan TC, Pearson ML, Silver LC, Jarvis WR. Guideline for prevention of surgical site infection, 1999. Hospital Infection Control Practices Advisory Committee. Infect Control Hosp Epidemiol. 1999;20(4):250-280 15 lerano C, Thursky K, Marshall C, et al. Appropriateness of Surgical Antimicrobial Prophylaxis Practices in Australia. JAMA Netw Open. 2019;2(11)

16 Tarchini G, Liau KH, Solomkin JS. Antimicrobial Stewardship in Surgery: Challenges and Opportunities. Clin Infect Dis. 2017;64(suppl_2):S112-S114 17 Leeds IL, Fabrizio A, Cosgrove SE, Wick EC. Treating Wisely: The Surgeon's Role in Antibiotic Stewardship. Ann Surg. 2017;265(5):871-873 
18 Murri R, de Belvis AG, Fantoni M, et al. Impact of antibiotic stewardship on perioperative antimicrobial prophylaxis. Int J Qual Health Care. 2016;28(4):502-507. 19 Plachouras D, Hopkins S. Antimicrobial stewardship: we know it works; time to make sure it is in place everywhere. Cochrane Database Syst Rev. 2017 20 Davey P, Marwick CA, Scott CL, et al. Interventions to improve antibiotic prescribing practices for hospital inpatients. Cochrane Database Syst Rev. 2017;2(2):CD003543. Published 2017 Feb 9

21 Nathwani D, Varghese D, Stephens J, Ansari W, Martin S, Charbonneau C. Value of hospital antimicrobial stewardship programs [ASPs]: a systematic review. Antimicrob Resist Infect Control. 2019;8:35. Published 2019 Feb 12. 


\section{Supplementary Files}

This is a list of supplementary files associated with this preprint. Click to download.

- supplement4.docx 